ISSN 1409-2441
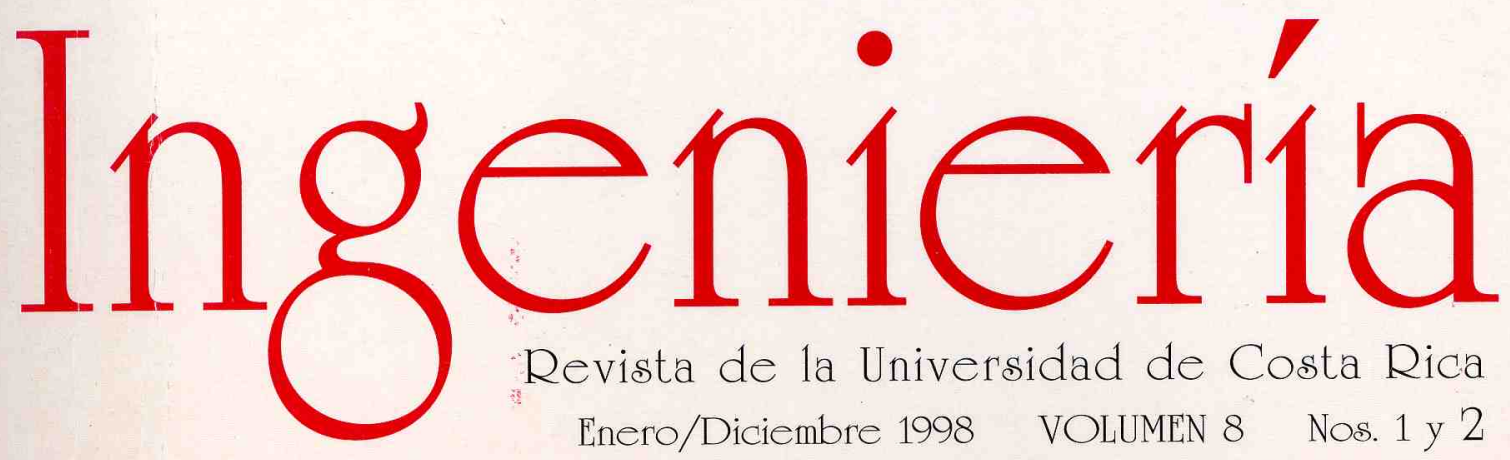

Revista de la Universidad de Costa Rica Enero/Diciembre 1998 VOLUMEN 8 Nos. 1 y 2 


\section{COMPUTACIÓN MÓVIL: UN PARADIGMA EMERGENTE}

Carlos Vargas C.J

\section{RESUMEN}

!Al computación móvil es un paradigma emergente que surge de la combinación de tres tecnologías: tenninales portátiles, comunicaciones inalámbricas, y redes de computadoras.En este articulo se introduc.:n los conceptos más relevantes de la computación móvil, ..nfatizándose lo referente a las tenninales portátiles. Además, se señalan varios de los nuevos problemas que atronta la ing.:nieria de sist.:nlas y el manejo de la movilidad en Intemet.

\section{SUMMARY}

Mobile computing is an emergent paradigm based on three tecnologíes: portable tenllinals, wireless conununications, and computer networks.This work introduces some relevant concepts about mobile computing, focusing on portable tenninals, pointing out several new problems tor the sotfWare engineering area, and Ihe managing of mobility on Intemet.

\section{INTRODUCCIÓN}

Computación móvil es un ténnino que enfatiza la capacidad de poder utilizar facilidades computacionales sin tener que estar atado a un sitio fijo, a lo que sí obligan las computadoras personales de escritorio y las estaciones de trabajo. Avances tecnológicos en el campo de la computación y de las comunicaciones han traído la capacidad de comunicación inalámbrica a los equipos portátiles, lo que le permite a un usuario conectarse a una red, incluso cuando éste se encuentre en movimiento. Ya comienza a hablarse de la oficina virtual $u$ oficina a distancia: "Un ordenador portátil, una tmjeta peMCrA, un teléfono móvil... Con ese equipa mento ya es posible disfrutar de las ventajas de la oficina a distancia" [ Sánchez, Y., 1996: Broida, R., 1998). También se habla de la oficina rodante [Bragi, D., 1999].

La meta de la computación móvil es "el acceso a la información, comunicación y servicios, en cualquier momento y en cualquier lugar" [Mazer, S. et al., 1995]. Dicha meta está apoyada por la combinación de tres elementos esenciales: 1) la infraestructura, compuesta de computadoras estacionarias y dispositivos de red; 2) computadoras portátiles, con propiedades fisicas y capacidades computacionales variables; y 3) canales de comunicación, alámbricos o inalámbricos, con anchos de banda variables, diferentes costos y varios niveles de confiabilidad.

Recientemente el mercado ha comenzado a ofrecer una gran variedad de equipos periféricos orientados, exclusivamente, hacia la computación móvil. La computadora portátil, pieza esencial de este paradigma emergente, es un término general que incluye un rango muy amplio de distintos tipos de unidades portátiles. Estas unidades poseén diferentes capacidades, tamaños, y formas que van desde las portátiles clásicas, pasando por las ultraportátiles y los dispositivos de palma que caben en una mano, hasta llegar a las diminutas tarjetas organizadoras que pueden llevarse en la billetera [Karagiannis, K., 1998]. 


\begin{tabular}{|c|c|}
\hline COMPONENTE & DESCRIPCIÓN \\
\hline Tillamook & $\begin{array}{l}\text { Nombre del primer procesador Pentium MMX que se produjo } \\
\text { con verdadera tecnología móvil. Se manufacturó con tecnolgía } \\
\text { de } 0.25 \text { micras en vez de } 0.35 \text { micras utilizada con } \\
\text { procesadores para pes de escritorio. }\end{array}$ \\
\hline MMO & $\begin{array}{l}\text { Tarjeta de procesador que constituye la tecnología móvil mas } \\
\text { reciente usada en el Pentium n. Esta incluye de manera } \\
\text { integrada la memoria caché de nivel } 2 \text {. }\end{array}$ \\
\hline TFT & $\begin{array}{l}\text { Tecnología de matriz activa para pantallas planas que logra } \\
\text { una imagen de mas alta calidad que la tecnolgía de matriz } \\
\text { pasiva. }\end{array}$ \\
\hline DSCN & Tecnología de matriz pasiva para pantallas planas. \\
\hline HPA & Es una tecnología superior a la DSCN pero inferior a la TFT. \\
\hline EDORAM & $\begin{array}{l}\text { Primera memoria principal diseñada para trabajar con equipos } \\
\text { basados en procesadores tipo Pentium. }\end{array}$ \\
\hline DRAM & Memoria mas veloz que la EDO. \\
\hline SDRAM & Memoria muy rápida, superior a la DRAM. \\
\hline USB & $\begin{array}{l}\text { Novedoso puerto que permite conectar docenas de dispositivos } \\
\text { periféricos (tales como cámaras digítales o de vídeo) en } \\
\text { cascada sin requerir energía eléctrica externa. }\end{array}$ \\
\hline Fast IR & $\begin{array}{l}\text { Puerto infrarojo rápido que pennite transmitir datos de modo } \\
\text { inalámbrico a una tasa de hasta } 4 \text { Megabits por segundo. }\end{array}$ \\
\hline PCMCIA & $\begin{array}{l}\text { También llamada tarjeta } \mathrm{Pe} \text { Permite expandir la } \\
\text { funcionalidad de una portátil. Existen tres tipos de tarjetas pe } \\
\text { denominados tipo 1, II Y III que se insertan en una ranura. } \\
\text { PCMCIA son las siglas de la organización que creó este } \\
\text { estándar. }\end{array}$ \\
\hline CardBus & $\begin{array}{l}\text { Un nuevo estandar mejorado de la tarjeta PC, con gasto de } \\
\text { corriente mas reducido y que opera con bus de } 32 \text { bits } \\
\text { alcanzando una transferencia de hasta } 600 \text { Megabytes por } \\
\text { segundo. }\end{array}$ \\
\hline ZV & $\begin{array}{l}\text { Sin requerir procesamiento del bus del sistema ni del } \\
\text { procesador principal, este nuevo estándar logra comunicación } \\
\text { directa entre el controlador de la tarjeta PC y los controladores } \\
\text { de vídeo y sonido, permitiendo correr videos sonoros en } \\
\text { pantalla completa sin pérdida de cuadros. }\end{array}$ \\
\hline Port Replicator & $\begin{array}{l}\text { Duplica los puertos permitiendo la conexión a múltiples } \\
\text { equipos periféricos sin necesidad de estar reconectando cables. }\end{array}$ \\
\hline Docking Station & $\begin{array}{l}\text { Dique de atraque que duplica los puertos y además incluye } \\
\text { bahías adicionales y ranuras de expansión. }\end{array}$ \\
\hline $\mathrm{NiMH}$ & $\begin{array}{l}\text { Un tipo de batería que no presenta el problema del "efecto de } \\
\text { memoria" pero que sí tiende a autodescargarse. }\end{array}$ \\
\hline Lithium-ion & $\begin{array}{l}\text { Un tipo de batería que no presenta el problema del "efecto de } \\
\text { memoria" ni tiende a descargarse. }\end{array}$ \\
\hline
\end{tabular}

Tabla $\mathrm{N}^{\circ}$ 1. Tecnicismos de la computadora portátil. 


\section{ESPECIFICACIONES TÉCNICAS.}

Tal como siempre acontece con la aparición de nuevas tecnologías, han surgido una gran cantidad de conceptos y ténninos nuevos importantes de conocer evaluar las capacidades funcionales que ofrecen los diversos equipos portátiles. Entre las tecnologías de punta se destacan las pantallas planas de matriz activa [Chinnock, C., 1998], los puertos infrarojos de alta velocidad [Mainelli, T., 1998], los procesadores móviles [Tsenter, B. y Golod, 1998]y las baterias de larga duración [Begun, D., 1998].

Normalmente, las revistas que anuncian las computadoras portátiles contienen alguna infonnación acerca de sus componentes. Internet ofrecela facilidad de tener acceso a los sitios de las compañías manufactureras de computadoras portátiles para obtener las caracteristicas técnicas de éstas con mucho detalle. Una comprensión clara de las especificaciones técnicas de una computadora portátil se facilita si se conoce la tecnología con que se manufacturaron los distintos componentes, junto con la nomenclatura que los identifica (ver tablaN $\left.{ }^{\circ} 1\right)$.

\section{UNA CLASIFICACIÓN DE LAS COMPUTADORASPORTÁTaES}

La selección de una computadora portátil, más allá de consideraciones netamente de costo, puede ser una tarea mucho más compleja que, por ejemplo, selecccionar una computadora personal de escritorio. Actualmente algunas computadoras portátiles ofrecen un desempeño muycercano al alcanzado por las computadoras personales de escritorio.

Establecer una línea divisoria absoluta que separe los distintos tipos de computadoras portátiles es prácticamente imposible. La tecnologíacambia tan rápidamente que lo que hoyse considera muy veloz mañana será lento. Sin embargo, es necesario contar con una clasificación para poder analizar y comparar las computadoras portátiles. Por ello, si aceptamos algún grado de traslape, podemos tipificar las computadoras portátiles atendiendo principalmente a atributos de peso, factores de forma, funcionalidad y precio, con independencia de marcas y modelos particulares [Vargas, C.,1999]. Obsérvese la figura $\mathrm{N}^{\circ} 1$.

Actualmente algunas computadoras portátiles ofrecen posibilidades muy cercanas a las computadoras personales de escritorio. Por ejemplo, las computadoras portátiles de alto nivel están diseñadas para que efectivamente sustituyan a las computadora;--personales de escritorio.

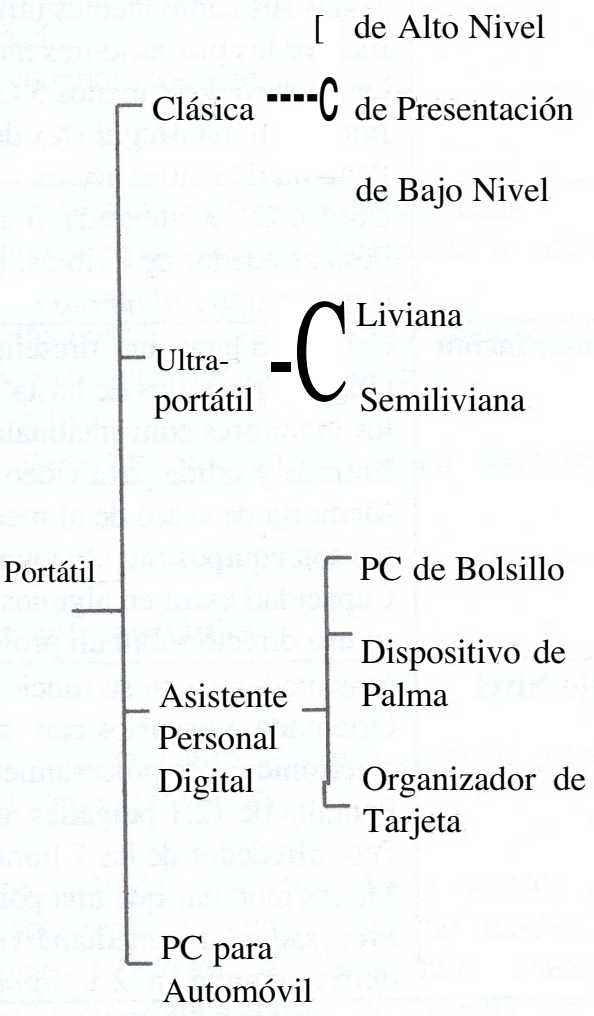

Figura 111/ 1. Una clasificación de las computadoras portátiles. 


\subsection{Portátil clásica (IIIptop)}

En la actualidad viene con procesadores Intel tipo Pentium, o equivalentes, manufacturados por otras empresas. Generalmente pesa entre $6 \mathrm{y}$ 11 libras, tiene $C D-R O M$ de IOX como mínimo, la pantalla de despliegue es de al menos 12.1 pulgadas en la diagonal. Usualmente posee 32 Megabytes de memoria principal y un disco duro de al menos 2.1 Gigabytes. Goza de al menos una ranura PCMCJA y un puerto infrarojo. Un mayor refinamiento distingue tres subcategorias: de alto nivel, de presentación y de valor.

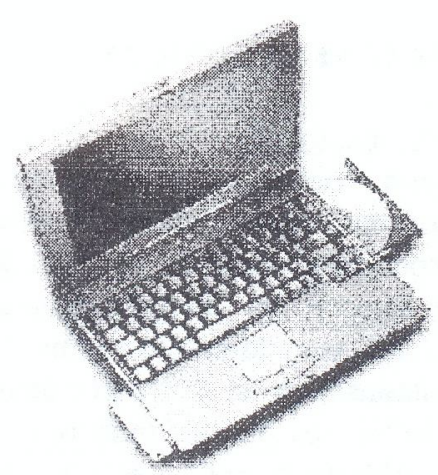

Figura $N^{\prime}$ 2. Computadora portátil. clásica (IIIptop)

\begin{tabular}{|c|c|}
\hline Portátil clásica & Características distintivas \\
\hline de Alto Nivel & $\begin{array}{l}\text { Diseñada para sustituir a la computadora personal. } \\
\text { Todos sus componentes utilizan tecnología de punta. } \\
\text { Incluye los procesadores móviles mas veloces. } \\
\text { Disco duro de al menos } 5 \text { Gigabytes. } \\
\text { Inicia con } 64 \text { Megabytes de memoria principal. } \\
\text { Pantalla de matriz activa. } \\
\text { Unidad DVD integrada, ó módulo opcional. } \\
\text { Peso alrededor de } 9 \text { libras, no muy liviana. } \\
\text { Baterías tino Lithium-ion. }\end{array}$ \\
\hline de Presentación & $\begin{array}{l}\text { Orientada hacia las presentaciones con multimedios. } \\
\text { Grandes pantallas de hasta } 15.1 \text {, que ofrecen un área visible competitiva con la de } \\
\text { los monitores convencionales. } \\
\text { Entrada y salida para video y sonido de tabla de ondas ( wffiJetable). } \\
\text { Memoria de video de al menos } 4 \text { Alegabytes. } \\
\text { No son equipos muy livianos y la batería tampoco tiene duración excesiva. } \\
\text { Capacidad extra en algunos modelos de poder soltar la cubierta de la pantalla para } \\
\text { su uso directo sobre un oroyector. }\end{array}$ \\
\hline de Bajo Nivel & $\begin{array}{l}\text { Mas moderada en su funcionalidad y desempeño que la portátil de alto nivel. } \\
\text { Orientada a usuarios con necesidades moderadas, tales como usuarios de correo } \\
\text { electrónico o de procesamiento de texto. El costo es bajo. } \\
\text { Pantalla de } 12.1 \text { pulgadas, no necesariamente de matriz activa. } \\
\text { Peso alrededor de las } 7 \text { libras. } \\
\text { Menos modular que una portátil de alto nivel. } \\
\text { Procesador de mediana velocidad, } 32 \text { Megabytes de memoria principal, disco } \\
\text { duro iniciando en } 2.1 \text { Giegabtes, y modem rápido. }\end{array}$ \\
\hline
\end{tabular}

Tabla $\mathrm{N}^{\circ} 2$. Computadoras portátiles clásicas. 


\subsection{UItraportátiles}

Son mucho mas livianas y mas delgadas que las computadoras portátiles de valor. Se distinguen dos clases: las livianas y las semilivianas. Ambos tipos utilizan procesadores Pentium y corren Windows 98.

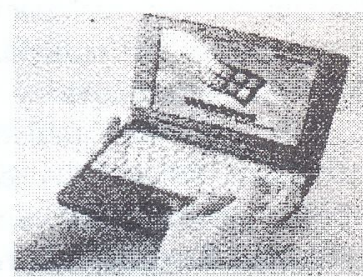

Figura\} $\measuredangle$ o 3. Computadora ultraportátil.

\begin{tabular}{|l|l|}
\hline Ultraportátil & Característica distintivas \\
\hline $\begin{array}{l}\text { Liviana } \\
\text { (Mininotebook) }\end{array}$ & $\begin{array}{l}\text { Tamaño muy compacto y poco peso, menos de } 3 \text { libras. } \\
\text { Pantalla de tamaño bastante reducido. } \\
\text { No poseé } C D-R O M \text { interno, aunque puede conectársele uno externo. } \\
\text { Teclado pequeño lo cual dificulta su uso. } \\
\text { Procesador de baja velocidad. } \\
\text { Disco duro y memoria de tamaños moderados. } \\
\text { Costo bajo. }\end{array}$ \\
\hline $\begin{array}{l}\text { Semiliviana } \\
\text { SubNotebook })\end{array}$ & $\begin{array}{l}\text { Extremadamente delgada, alrededor de 1.8 pulgadas de grosor. } \\
\text { Equipo muy poderoso altamente portable que incorporan lo último en } \\
\text { tecnología. } \\
\text { Bastante mas liviana que una portátil de Valor, aunque ligeramente mas } \\
\text { pesada que una portátil liviana. Entre } 3 \text { y } 5 \text { libras de peso. } \\
\text { Pantalla grande de matriz activa. } \\
\text { Procesador muy veloz, abundante memoria y disco duro de alta } \\
\text { capacidad } \\
\text { Costo muy elevado. }\end{array}$ \\
\hline
\end{tabular}

Tabla $N^{\circ}$ 3. Computadoras ultraportátiles.

\subsection{Asistentes personales digitales}

También llamados PDAs por sus siglas en inglés (Personal Digital Asistant). Son extremadamente compactos y livianos. A lo sumo alcanzan las 2 libras y algunos únicamente pesan 2 onzas. A diferencia de las portátiles clásicas o de las ultraportátiles que ejecutan Windows 98, éstas máquinas t:iecutan una versión reducida de Windows, llamada Windows
$C E$, u algún otro sistema operativo patentado, tal como el Palm OS.

Sus pantallas son reducidas y carecen de los dispositivos de almacenamiento masivo, tales como CD-ROM o disco duro. Dadas sus pequeñas dimensiones, la entrada de datos siempre resulta bastante dificultuosa. Son diseñadas para complementar a una computadora personal o a una portátil clásica, no para sustituirlas. La fonna de intercambio de 
información es típicamente a través del puerto infrarojo. Pese a sus capacidades reducidas, resultan ideales para ciertas aplicaciones y su costo es bajo. La mayoria de las unidades operan con baterias alcalinas. AA ó AAA, con duraciones que van desde un dia hasta varias semanas. Algunas comienzan a utilizar baterias recargables de NiMH y más recientemente las Lithium Jon.

Atendiendo a sus capacidads y tamaño se distinguen tres tipos de unidades. La mayor es la llamada PC de bolsillo, o Handheld en inglés, que pesa menos de 2 libras. La intermedia es el dispositivo de palma o Palm-sized en inglés, que pesa menos de 8 onzas. Y mas diminuto aún es el organizador de taJ:jeta PC que únicamente pesa 2 onzas y puede portarse dentro de la billetera.

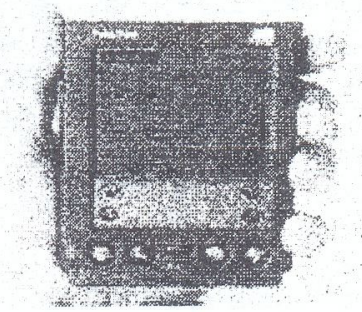

Figura lv.04. Dispositivo de palma.

\begin{tabular}{|l|l|}
\hline Asistente personal & Característica distintivas \\
\hline PC de Bolsillo & $\begin{array}{l}\text { Muy pequeña y liviana. Pesa entre } 12 \text { y } 24 \text { onzas con un tamaño típico } \\
\text { alrededor de } 1.3 \times 7.5 \times 4.0 \text { pulgadas, aún menor si es monocromática. } \\
\text { Incluye capacidad de grabación de voz y pantalla de tacto. } \\
\text { Ejecuta Windows CE u otros sistemas operativos patentados. } \\
\text { Teclado integrado pero cuyo uso resulta dificil debido al tamaño reducido. } \\
\text { Típicamente viene con un puerto serial y otro infrarojo, una memoria entre } \\
\text { y } 32 \text { Megabytes y procesadores corriendo entre } 60 \text { y 100 Mhz. } \\
\text { Tiene un modem interno y soporte a tarjetas pe tipos I y II que le extiende la } \\
\text { funcionalidad. tal como conectársele una cámara digital. } \\
\text { Algunas vienen con un diaue de atraaue. }\end{array}$ \\
\hline Dispositivo de Palma & $\begin{array}{l}\text { Tan pequeño y liviano que puede portarse en el bolsillo. } \\
\text { Pesa entre } 5 \text { y } 7 \text { onzas y mide alrededor de } 0.7 \text { x 3.2 x 4.7 pulgadas. } \\
\text { Ejecuta un sistema operativo patentado o una versión de ll/indows CE. } \\
\text { Viene con } 8 \text { lvlegabytes o menos de memoria pero soporta tarjetas de } \\
\text { memoria CompactFlash para almacenamiento ex1ra. } \\
\text { Carece de teclado, pero los datos pueden entrarse vía la pantalla de tacto. } \\
\text { Fácilmente se sincronizan con la computadora personal a través del puerto } \\
\text { infrarojo. Contiene un modem rápido y ofrece el servicio del correo } \\
\text { electrónico. }\end{array}$ \\
\hline $\begin{array}{l}\text { Unidad muy diminuta que puede ser llevada en la billetera. } \\
\text { Pesa menos de } 2 \text { onzas y su tamaño es alrededor de 0.3 x 3.8 x 2.1 pulgadas. } \\
\text { Los datos se deben capturar desde otra computadora, por cuanto usualmente } \\
\text { son unidades solo de lectura. } \\
\text { La vida de la batería es bastante larga y ejecuta un sistema operativo } \\
\text { patentado. }\end{array}$ \\
\hline de Tarjeta PC
\end{tabular}

Tabla $N^{\circ}$ 4. Asistentes personales digitales 


\subsection{PC para Automóvil}

Novedoso equipo diseñado para constituirse en un elemento integrador del automóvil, ya que controla los teléfonos celulares, el sonido, los sistemas de seguridad, y otros sistemas. Ofrece una amplia gama de funciones. Por ejemplo, responder a comandos de voz e integración con dispositivos de posicionamiento global (GPS) para establecer un mapa de la ruta a seguir.

Las AutoPC son las primeras unidades de Ciaríon Corporation. Estas vienen con controles activados por voz capaces de reconocer un vocabulario de hasta 200 palabras. Penniten intercambio de datos con un asistente personal digital a través del puerto infrarojo. Intel también está desarrollando un producto similar denominado Connected Car, mientras que la empresa Chrysler optó por utilizar tecnología existente: cualquier PC de bolsillo que ejecute Windows CE conjuntamente con un programa desarrollado por Motoro/a.

\section{PROBLEMAS DE LOS AMBIENTES MÓVILES}

El poder conectar una computadora portátil a una red, sea ésta inalámbrica o estacionaria, incrementa enonnemente los servicios y aplicaciones disponibles al usuario, tales como correo electrónico o transferencia de archivos desde sitios remotos. Sin embargo, la introducción de móvilidad y comunicaciones inalámbricas acarrea algunos retos técnicos a la ingenieria de sistemas.

Dado que las computadoras son típicamente configuradas para utilizarse en un sitio fIjo, en algunas situaciones la incorporación de la computación móvil obliga a los diseñadores de sistemasa adaptar las estructuras de los sistemas tradicionales. Otras veces, el diseñador deberá afrontar problemas completamente diferentes de aquellospropios de las redes estacionarias.
La portabilidad induce algunas restricciones que no se presentan en las computadoras fijas. Por ejemplo, las computadoras mÓvilesusan baterias lo que representa una restricción no encontrada en las computadoras fIjas. Cuando se produzca una condición de batería baja, entonces podria cambiarse de una interfase gráfica a una basada en texto, consumiendo así menos energía.

La restricción impuesta por el tamaño de algunas computadoras portátiles obliga a consideraciones especiales en el diseño de la interfase de usuario [Welling, G. y Badrinath, B., 1997]. Cuando la pantalla de despliegue sea muy pequeña, como acontece con los asistentes personales digitales, el diseñador de la aplicación deberá tomar en cuenta que quizá no disponga de un ambiente de ventanas. Algunas tenninales portátiles, aunque pequeñas, sí soportan las ventanas. Sin embargo, deberá considerarse que resulta impráctico mantener varias ventanas simultáneamente, por cuanto resulta dificil localizarlas cuando se hayan apiladas unas sobre otras en varios niveles de profundidad. También deberá tomarse en cuenta que los elementos típicos encontrados en las ventanas, tales como los bordes y la barra del título, consumen un espacio valioso de la pantalla.

euando una aplicación que administra el correo electrónico, detecta que la conección se encuentra en un estado intermitente, entonces podria escojer el mantener en memoria los mensajes de salida, enviándolos luego, cuando detecte suficiente ancho de banda. Si la situación restrictiva perdura, entonces podria entrarse a un estado iconizado, como sugerencia para inhibir la actividad de la red.

Como norma, una aplicación en un ambiente de computación móvil debería dinámicamente mejorar su calidad de servicio cuando un recurso está disponible, y paulatinamente degradarse cuando se comience a carecer del mismo. Entre los recursos que deben ser considerados se incluyen: la bateria, la memoria, el procesador, y la red [Lorch, J. y Smith, A, 1998]. 
Forman y ZahOljan [Forman, G. y Zahorjan, 1., 1994] señalan algunos de los retos pertinentes a la computación móvil y a las comunicaciones inalámbricas. Los problemas inherentes a la computación móvil son diversos y se manifiestan en las diferentes capas que definen la arquitectura de la red.

A nivel de capa fisica, los mayores problemas enfrentados por las comunicaciones inalámbricas, en contraste con las comunicaciones alámbricas, provienen de que la señal es perturbada por el ambiente. Los caminos que la señal recorre pueden quedar bloqueados, o la señal es perturbada por el exceso de "ruido". En consecuencia, ocurren desconecciones con mucha más frecuencia que con las comunicaciones alámbricas. De modo que si un sistema depende mucho de la red entonces dejará de funcionar durante las fallas de ésta.

Una mayor autonomía de la térnÚnal móvil implica una mejor tolerancia hacia la posibilidad de desconección de la red. Ciertas aplicaciones podrian correr localmente en la terminal móvil en vez de fragmentarse a través de la red. Conviene explotarse que las computadoras ligeras actualmente gozan tanto de alta portabilidad como de gran autonomía. El ingeniero de sistemas deberá decidir como gastar los recursos de la red: previniendo que ocurran las desconecciones $\mathrm{o}$ buscando establecer sistemas que enfrenten el problema de las desconecciones más apropiadamente. Si las desconecciones ocurren con duraciones muy cortas, la operación asincrónica puede ocultarlas. El $X$-Windows usa esta técnica para mejorar el rendimiento del sistema.

Otro problema con que se topan las comunicaciones inalámbricas es el bajo ancho de banda [Halsall, F., 1998]. Mientras que, por ejemplo, éstas apenas logran alrededor de un Megabit por segundo con tecnología infraroja, y de dos Alegabits con radio comunicaciones, con fast Ethernet en redes no inalámbricas se alcanzan los cien Megabits. De ahí que se requieran ciertas técnicas de software que manejen esta situación. Las técnicas de comprensión con buffer mejoran el rendimiento ya que, usualmente, bloques mayores producen tasas de compresión mas altas.

A nivel de capa de red, la que se encarga de encanÚnar los mensajes asociados a las capas de protocolo superior a través de toda la red, surge una complicación adicional para poner en ruta un mensaje a un anfitrion móvil: la red deberá primeramente localizarlo.

En la siguiente sección se examina como este problema se resuelve a nivel de Internet.

\section{COMPUTACiÓN MÓVIL EN EL CONTEXTO DE LA RED INTERNET}

La meta de poder accesar infonnación en cualquier momento y desde cualquier lugar es perseguida tanto por la computación móvil, como por Internet. Usuarios buscando acceso a Internet desde sus tenninales móviles, requieren que éstas puedan conectarse a Internet y que se mantengan así, aún cuando ellos estén moviéndose de un lugar a otro, estableciendo nuevos enlaces y alejándose de enlaces previamente establecidos.

Desafortunadamente, cuando el protocolo de Internet (IP) fue diseñado no se consideró a los usuarios móviles. El supuesto fue que las conecciones se harian mediante enlaces fijos. El esquema de direccionamiento mediante el IP no ayuda a la computación móvil. Primero entendamos cómo son usadas las direcciones en Internet [Perkins, E., 1997]. Por una parte, el protocolo de control de la trasmisión (TCP) mantiene el seguimiento del estado de la sesión interna entre los nodos finales en comunicación a través de la dirección IP de dichos nodos, que es almacenada junto con los números de puertos, ya que estos últimos son los selectores para el demultíplexamiento de la sesión. Es decir, que el TCP usa la dirección IP para identificar. Por otra parte, las direcciones IP también son 
utilizadas para encontrar una ruta entre los nodos finales. La ruta de ida no necesariamente tiene que ser la misma de vuelta.

En relación con las tenninales móviles, el esquema de direccionamiento conduciría a que se debe tener una dirección IP estable para que la tenninal sea identificable por otros computadores de la red Internet, pero entonces el enrutamiento también seria estable, significando que los paquetes siempre se dirigirían al mismo lugar, lo cual no resulta en

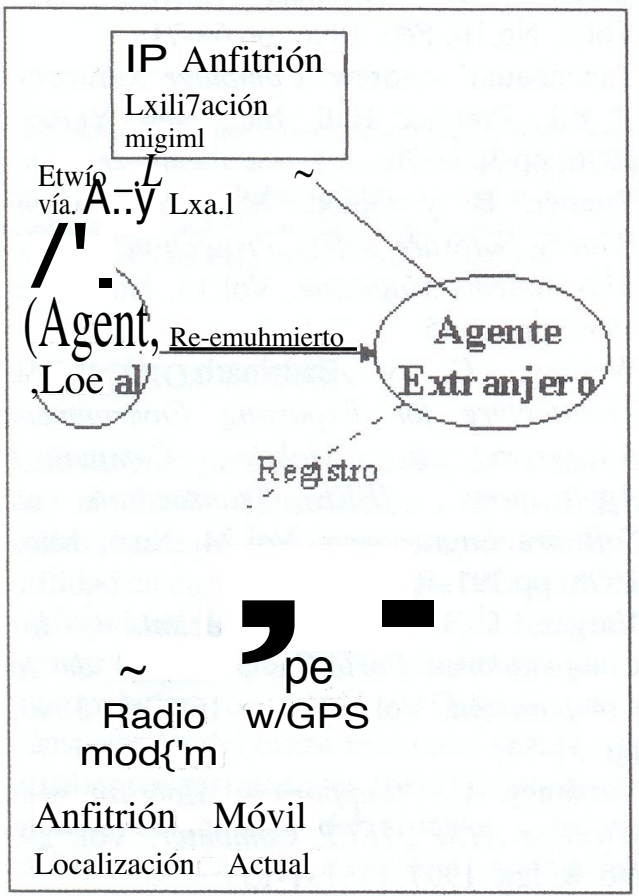

movilidad.

Figura IV" 5. Envío de paquetes con IP Móvil.

Debido a la situación anterior, la IETF (Internet Engineering Task Force) compuesta por mas de 60 grupos de trabajo, se dió a la tarea de fonnular la especificación del protocolo llamado IP móvil [Perkins, E., 1996]. Este protocolo es una versión que extiende el IP para que soporte la computación móvil en la red Internet, ya que pennite utilizar dos direcciones IP, una para identificación y la otra para enrutamiento.

El problema de localizar un anfitrión móvil, se basa en un proceso de enrutamiento que utiliza agentes de dos tipos, denominados agentes extranjeros y agentes locales, quienes cooperan para remitirle paquetes al anfitrión desde su dirección base al moverse de un área a otra [Tanenbaum, A., 1996].

Conceptualmente el proceso cooperativo se lleva a cabo como se describe a continuación [Varshney, U., 1997]. El designado agente local de un anfitrión móvil remite paquetes desde la localización original del anfitrión hacia su localización actual. En la medida en que el anfitrión se está moviendo, éste se registra con un agente extranjero quien sirve al área siendo visitada por el anfitrión. Primeramente el agente extranjero notifica al agente local del anfitrión, luego registra al anfitrión y le em:ía al agente local la infonnación del registro. Cualquier agente extranjero previo es también notificado cuando un anfitrión se mueve. Nótese que los paquetes continuan siendo enviados a la localidad previa del anfitrión, donde el agente local los copia y los remite al agente extranjero actual, quien a su vez los envía al anfitrión móvil.

\section{CONCLUSIONES}

Alguién mencionó, que la historia recordaria al periodo de 1996 al 2006 como "la década de la computación móvil". Quizá esta aseveración sea muy osada. Sin embargo, al observar las tendencias se puede anticipar que la computación móvil junto con Internet jugarán un papel importante en la sociedad mundial.

La ingeniería de sistemas ha comenzado ya a jugar un papel constructivo en el desarrollo de estas dos tecnologías recientes. La ingenieria de sistemas está poniendo mucha atención a la aplicación de métodos y principios orientados 
hacia el desarrollo de sistemas, cuyos componentes exhiban alguna fOffila de movilidad a través de redes y requiera conocimiento acerca del dominio dentro del cual el movimiento tenga lugar.

Pese a que la computación móvil no ha alcanzado una madurez completa, ya se cuenta con un fabuloso desarrollo en las transmisiones vía satelite que ha logrado una cobertura mundial en transmisiones inalámbricas. Tambíen existe un gran interés en lograr incorporar masivamente los servicios de multimedia al ambiente de la computación móvil.

\section{BffiUOGRAFÍA}

1. Begun, D., "Mobile Computing", Computer Shopper, Vo1.20, No.4, May. 1998, pp.219234.

2. Bragi, D., "Road Stories", Portable Computing, Vo1.2, No.12, Jan. 1999, pp. 128-129.

3. Broida, R., ' 4ssembling the Ideal Mobile Office", Movile Computing \& Communications, Vol.9, No.7, Julio 1998, pp. 107-115.

4. Chinnock, C., "Small and Alid-Size FlatPanel Displays Heat Up the Afainstream", Electronic Design, Vol.46, No.19,Ago. 1998.

5. Fonnan, G. y ZahOljan, 1., "The Challenges of Mobile Computing", IEEE Computer. Vol. 7, No. 4, 1994, pp.38-47.

6. Halsall, Fred. Comunicacion de Datos, Redes de Computadores y Sistemas Abiertos. $\quad 4^{\mathrm{a}} \quad$ Ed., Addison-Wesley Iberoamericana, S.A.: Venezuela, 1998.

7. Karagiannis, K., "Notebook Technology Watch", Laptop, V01.1 NO.9, Sep. 1998, pp. 128-135.

8. Lorch, 1. y Smith, A., 1998, "Software Strategies for Portable Computer Energy Managemenf', IEEE Personnal Communications, Vol. 5, No. 3, 1998, pp, 60-73.
9. Mainelli, T., "Invisible Connections: Infrared \& Portable Computers", Guide to Upgrading, Vo1.6, No.2, Feb. 1998, pp.9798.

10. Mazer, S. et al. "Issues in lv/obile Computing Systems", IEEE Personal Communications, Vo1.2, No.6, Dic. 1995.

11. Perkins, E., "Mobile IP", IEEE Communications Magazine, Vol. 35, No. 5 , May. 1997, pp.84-99.

12. Perkins, E., "Ipv4 Mobility Supporf', BFe 2002. Oct. 1996.

13. Sánchez, V., "Oficinas Móviles", Net, Yo1.3, NO.16, Feb. 1996, pp.68-71.

14. Tanenbaum, Andrew. Computer Networks. $3^{\mathrm{a}}$ Ed., Prentice Hall, Inc.: New Yersey, 1996, pp.367-370.

15. Tsenter, B. y Golod, M, "Lithium-Ion Battery Software safety Protection", IEEE AES Systems Magazine, V01.13, No.9, Set. 1998, pp.23-25.

16. Welling, G. y Badrinath, B., "An Architecture iór Exporting Environment Awareness to Mobile Computting Applications", IEEE Transactions o/1 Software Engineering, Vol. 24, No.5, May. 1998, pp.391-400.

17. Vargas, C., "Una clasificación de las Computadoras Portátiles", I\undo de la Computación, Vol. 13, No. 16, Feb. 1999, pp. 51-54.

18. Yarshney, u., "Supporting Mobility with Wireless ATM', IEEE Computer, Vol. 29, No. 8, Jan. 1997, PP. 131-133. 